\title{
Very early processing of emotional words revealed in temporoparietal junctions of both hemispheres by EEG and TMS
}

\author{
Vincent Rochas • Tonia A. Rihs • Nadia Rosenberg • \\ Theodor Landis · Christoph M. Michel
}

Received: 27 August 2013 / Accepted: 10 January 2014 / Published online: 5 February 2014

(C) Springer-Verlag Berlin Heidelberg 2014

\begin{abstract}
We investigate the contribution of both hemispheres in a lateralised lexical decision paradigm with emotional and neutral words in healthy volunteers. In a first experiment, high-density EEG analysis using source imaging methods revealed early specific participation of the temporoparietal junctions (TPJ) in both hemispheres for the detection of words. Then, in an event-related transcranial magnetic stimulation experiment with the same task, the disruption of left or right TPJ compared with a control stimulation over the vertex showed a slowing that is more pronounced when words are emotional and presented in the left visual field (LVF). This indicates that interference with both left and right TPJ results in impaired processing of words that were presented to the LVF. In addition, these results point to a specific cooperative contribution of the right hemisphere in the processing of words with emotional content compared with neutral words at very early stages. Results from the two experiments can be integrated in a brain-based spatiotemporal model of the early detection of written words.
\end{abstract}

Keywords Lexical decision - Word detection - Emotion . Lateralisation · Right hemisphere · EEG · Source imaging · TMS

V. Rochas $(\bowtie) \cdot$ T. A. Rihs $\cdot$ N. Rosenberg $\cdot$ T. Landis .

C. M. Michel

Functional Brain Mapping Laboratory, Department

of Fundamental Neuroscience, CMU, University of Geneva,

Rue Michel-Servet 1, 1206 Geneva, Switzerland

e-mail: vincent.rochas@unige.ch

\section{Introduction}

Since the seminal works of Broca (1861) and Wernicke (1874), the left cerebral hemisphere in right handers is known to be dominant for language processing. However, the study of split-brain patients with surgically disconnected hemispheres (Sperry 1961; Gazzaniga and Sperry 1967; Gazzaniga and Hillyard 1971; Gazzaniga 2005 for a review) and the study of healthy subjects with lateralised stimulus presentations (for a review see Springer and Deutsch 2004) leave no doubt that the right hemisphere also plays a considerable role in language processing. More recently, neuropsychological studies, lesion-based studies, and imagery studies (Stroobant et al. 2009; Lidzba et al. 2011; Mohr et al. 2007) revealed the participation of the right hemisphere in various features of language processing-e.g. intonation (Ross et al. 1988; Pell 2007), concreteness (Papagno et al. 2009), or context-related language (Diaz and Hogstrom 2011). Most classical is the relation of the right hemisphere with the emotional dimension of language processing. The special role that emotional words play with respect to cerebral laterality has been recently summarised (Landis 2006).

The present study aims to assess language lateralisation and the interaction between the two hemispheres in relation to the treatment of the emotional content of the words. It is based on earlier work from our group (Ortigue et al. 2004) that used a lexical decision task with a simultaneous bilateral presentation of letter strings. In this previous work, event-related potential (ERP) analysis showed a distinct scalp topography for emotional words presented in the right visual field (RVF), indicative of a specific intracranial generator configuration for these words. The well-known emotional word advantage was accompanied by increased activation of right lateral occipital brain areas at around 
100-140 ms. However, somewhat unexpectedly, this effect was only found for emotional words presented to the RVF and not to left visual field (LVF), despite the enhanced performance for emotional words in both conditions.

Derived from the study of Ortigue et al. (2004), the present study does not only better control the stimulus material and improve the analysis of the ERPs, but also assesses the early contribution of the right hemisphere to emotional word processing by applying transcranial magnetic stimulation (TMS). We performed a first experiment where we tracked the early brain processes of real word detection by high-density electroencephalography (256-channel EEG) using statistical analysis of the ERPs in the sensor space (voltage topography) and source space [electrical source imaging (ESI)] (Michel and Murray 2011). In the second experiment on an independent group of participants, eventrelated TMS was alternatively delivered in two regions that were identified in the first experiment to be recruited in these early stages of word processing - the left and right temporoparietal junctions (TPJ). The time window of stimulation was also selected on the basis of the first experiment. Behavioural measurements after interruption of these areas allowed to evaluate their implication in word processing.

The combination of these two time-resolved brain imagery methods allows a comprehensive understanding of particular actors and processes of the network in a given cognitive task. Our findings are then discussed and included in a functional spatiotemporal model of early processing of written words and emotionality.

\section{Materials and methods}

\section{Study 1 (EEG)}

\section{Participants}

Eighteen healthy volunteers participated in this study approved by the Ethical Committee of the University Hospital of Geneva, Switzerland. Included participants were young adult males, right-handed, aged from 20 to 32 years (mean age $=24.8 ; \mathrm{SD}=3.5$ ). All had normal or correctedto-normal visual acuity and French as their mother tongue. None had experienced psychiatric or neurological disease, or any language difficulties (e.g. dyslexia) in their past. Participants were paid for their participation $(30 \mathrm{CHF} / \mathrm{h})$. Experiments were performed with the informed and overt consent of each participant.

Three participants were excluded after the training session and before any EEG recording, due to low detection rates in at least one condition. In addition, the recordings of two participants had bad impedances or muscular artefacts during the EEG acquisition and were also excluded from further analysis. The analyses for study 1 were thus conducted with thirteen participants.

\section{Stimuli and procedure}

A lexical decision task was used in a simultaneous bilateral presentation. This is a revised version of the task previously investigated in our laboratory (Ortigue et al. 2004; Mohr et al. 2005). On the screen, at 1 meter from the participant, two strings of letters were displayed at the same time on each side of a central fixation cross, in the LVF and in the RVF. Letter strings represented either a real French word or a pronounceable pseudo-word. Two kinds of words were used: neutral and emotional words. This resulted in five possible stimulus conditions: a neutral word in the LVF, a neutral word in the RVF, an emotional word in the LVF, an emotional word in the RVF (all paired with a pseudo-word in the opposite side), or two pseudo-words. All letter strings were constituted of four black letters displayed in upper case $(1.1 \mathrm{~cm}$ high and $4.2 \mathrm{~cm}$ length) on a white background. The letter strings were presented from $2.00^{\circ}$ to $4.85^{\circ}$ of visual angle from the fixation point on each side. Thus, the two-letter strings were simultaneously displayed in distinct horizontal visual hemifields. The presentation time was $13 \mathrm{~ms}$. During the experiment, the participants were asked to respond during the $2 \mathrm{~s}$ following the stimulus if they had seen a word or not. If yes, they had to press a button with the hand on the side on which they detected the word. No response was required if they did not detect a word on either side. The inter-stimulus interval was randomly jittered between 2,300 and 2,600 ms. A sample trial is shown in Fig. 1.

Four-letter substantives with highest frequencies (occurrences by million determined from a written database of text book material and an oral database of movie subtitle material) were taken from the freely accessible and well-controlled database for the French language "Lexique 3.55" (New et al. 2001; see also http://www.lexique. org). Independent participants $(n=12)$ rated these words for familiarity, concreteness, imageability, emotional neutrality, and emotionality on a 1-9 Likert scale. Only fourletter words with high familiarity, low concreteness, and low imageability were kept. The 16 words with highest neutral and lowest emotional ratings were chosen for the neutral category (e.g. the French word "voie"), while the reverse-i.e. highest emotional, lowest neutral ratingswere included in the emotional category (e.g. the word "peur"). The selected words were matched with pronounceable pseudo-words with identical patterns of consonants and vowels with four letters. Pseudo-words with spelling close to familiar foreign language words (commonly English and German) were excluded to avoid any interaction in potential multilingual participants. $t$ tests on the selected 

milliseconds (ms) of a trial for the presentation of one pair of letter strings in the lexical decision task in study 1 (EEG) and study 2 (TMS)
Fig. 1 Schematic chronology in

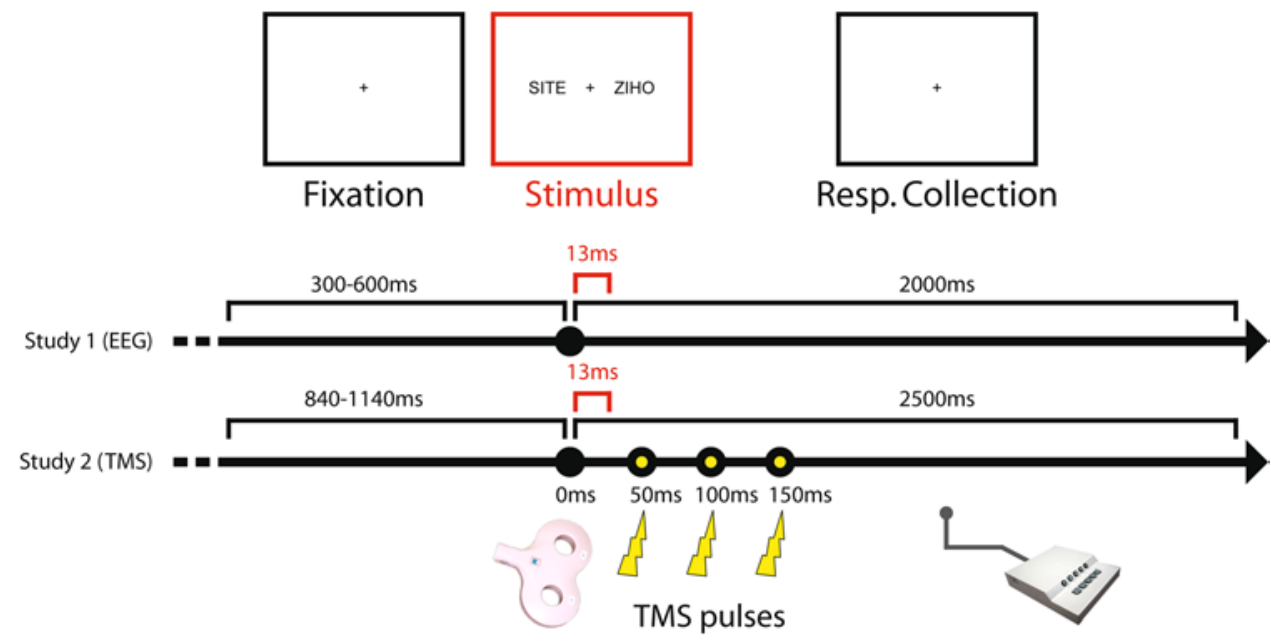

EEG data acquisition and preanalysis

words showed that neutral and emotional words did not differ in terms of frequency (respective averages 92.25 vs. 95.44; with a $t=-0.73$ and $p=0.89$ ), familiarity (7.40 vs. $7.80 ; t=-1.88$ and $p=0.07$ ), concreteness (3.7 vs. 3.28; $t=0.85$ and $p=0.40$ ), or imageability (4.63 vs. 3.94; $t=-1.24$ and $p=0.23$ ).

The task was divided into 8 blocks ( 4 min each) of 96 trials fully randomised for each block and for each participant. The total number of repetitions was 128 for neutral words in the LVF, 128 for neutral words in the RVF, 128 for emotional words in the LVF, 128 for emotional words in the RVF, and 256 for two pseudo-words in both hemifields.

To avoid learning effects during the EEG recording due to the repeated presentation of the words, the subject practised the task in four blocks the day before the EEG recording. Before each EEG session, the whole set of words were presented individually in a central position below the fixation point and the participants performed a simple lexical decision task without EEG recordings. This learning session was performed to further avoid learning effects during task performance.

\section{Behavioural data}

Average accuracies and median reaction times (RT) were calculated for each condition in each subject. These behavioural data were plotted with their standard errors in bar graphs (see Fig. 2). The statistical analysis of the behavioural data was performed to replicate previous studies and was thus based on the prediction that words are better detected when presented in the RVF than to the LVF (Young et al. 1980; Graves et al. 1981; Iacoboni and Zaidel 1996; Babkoff et al. 1997) and that emotional words are better detected than neutral words (Landis, 2006; Schacht and Sommer 2009; Kissler and Koessler 2011; Skrandies 2013). Missed events and false alarms were also computed in order to detect potential outlier subjects (not represented).
High-density EEG data were collected during 8 blocks of the task. The system used for acquisition was a 256-channel (AgCl carbon-fibre-coated electrodes) HydroCel Geodesic Sensor Net in association with Net Station EEG acquisition software (Electrical Geodesics, Inc., Eugene, OR, USA). Recordings were referenced to the vertex $(\mathrm{Cz})$ and sampled with a 16-bit analog-to-digital converter at $1,000 \mathrm{~Hz}$ with a DC removal. All electrode impedances were kept below $30 \mathrm{k} \Omega$.

Offline analyses of the EEG were conducted using the "Cartool" software (D. Brunet, Functional Brain Mapping Laboratory and CIBM, Geneva, Switzerland; http:// sites.google.com/site/fbmlab/cartool). The signals of peripheral electrodes (on the cheeks and the nape) were omitted from the 256 channels, and the remaining 204 channels were submitted to further analysis. The EEG of these remaining channels was bandpass filtered from 0.16 to $40 \mathrm{~Hz}$ and converted to the average reference. The continuous EEG was visually inspected, and only epochs without eye blinks or muscular artefacts and with correct responses were taken into account. The number of averaged epochs was counterbalanced between the four conditions for each participant-i.e. we randomly picked a number of epochs determined by the condition with the lowest number of accepted epochs for each individual (average number of accepted epochs 57.2 $\pm 6.4 \mathrm{SE}$ ). Therefore, for a given subject, the different conditions included comparable signal-to-noise ratios. The remaining epochs were averaged by condition from 0 to $300 \mathrm{~ms}$ after the onset of the stimulus to reveal the event-related potential (ERP) for each participant. For each individual, artefactual electrode signals were interpolated using a spherical spline interpolation (Perrin et al. 1987). All individual ERP's were realigned on the peak of the global field power of the P100 component determined once for each subject to reduce 
the inter-individual variability of the time for visual information transfer from the retina to primary visual cortex (Woody 1967; Morand et al. 2000). Realignment has been -27 up to $+30 \mathrm{~ms}$ from the original onset of the stimulus. As visual information does not reach the primary visual cortex before $50 \mathrm{~ms}$ (Alexander and Wright 2006; Thorpe 2009), this realignment on the first milliseconds does not impede the analysis of cognitive cortical processes but reduces their temporal smearing. Finally, ERPs were downsampled to $500 \mathrm{~Hz}$ before statistical analysis.

\section{ERP's topographic ANOVA}

Since the aim of this first experiment was to determine when and where in the brain the different stimuli were processed to guide the subsequent TMS study, the analysis of the high-density ERPs was entirely based on topographical analysis of the scalp potential fields and on ESI. In contrast to the conventional waveform analysis, the topographic analysis gives non-ambiguous and reference-free information about changes in the underlying generators in time and between conditions (Michel et al. 2009; Michel and Murray 2011; Murray et al. 2008).

The first step of the analysis consisted in a topographic analysis of variance ( $T$-ANOVA) based on paired permutation tests with entire topographies as dependent variables (Murray et al. 2008; Koenig et al. 2013). This analysis was performed with the freely available software randomisation graphical user interface (RAGU; Koenig et al. 2011), which allows for multivariate $T$-ANOVAs. The program calculates the global field power of the normalised difference maps between conditions for each subject, also called Global Dissimilarity (Lehmann and Skrandies 1980; Murray et al. 2008). The Dissimilarity is calculated repeatedly after random shuffling the condition assignments of the maps across subjects. This permutation statistic enables us to compare the real distribution of the Map Dissimilarity against the random distribution. The significance of the effect is then determined by the percent of randomly obtained values of the Dissimilarity that are larger than the real value. These $T$-ANOVAs were performed for each individual time point from 50 to $250 \mathrm{~ms}$ post-stimulus in a two-by-two withinsubject factorial design ( $p$ value $<0.05 ; 5,000$ repetitions); for words detected in the LVF versus in the RVF (Laterality); and for detected neutral words versus emotional words (Emotionality).

\section{Electrical source imaging (ESI)}

In order to estimate the location of current generators in the brain, the local auto-regressive average (LAURA) algorithm, a distributed linear inverse solution comprising biophysical laws as constraints (Grave de Peralta Menendez et al. 2001, 2004; Michel et al. 2004 for review), was used. Source estimations were computed from ERPs of each participant for each condition according to the factors Emotionality and Laterality using the Cartool software in a space of 3,005 solution points restricted to the grey matter of the MNI brain (Spinelli et al. 2000; Brunet et al. 2011). For each participant and each condition, a normalisation of the estimated activity values was performed by $z$-score transformation of the activity at each solution point for a given time point. The transformation in $z$-scores homogenised inter-individual contrasts by removing the background noise and reducing extreme values. Using these $z$-scores, as dependent variables, time-point-by-time-point ANOVAs were computed for each solution point $(p<0.01$ for at least five consecutive time frames corresponding to $10 \mathrm{~ms}$ and with a size restriction criterion of five neighboured solution points) with the STEN toolbox (STEN toolbox developed by J.-F. Knebel, Functional Electrical Neuroimaging Laboratory, Lausanne, Switzerland. www.u nil.ch/fenl/Sten). Laterality and Emotionality of the words were taken as within-subject factors. Post hoc analyses, consisting in $t$ tests on each solution point for each time frame, were computed to define the direction of the significant differences established by the ANOVA.

\section{Study 2 (TMS)}

\section{Participants}

Thirteen healthy volunteers participated in this study approved by the Ethical Committee of the University Hospital of Geneva, Switzerland. These participants did not take part in study 1 (EEG). They were young adult males, right-handed, aged from 21 to 33 years old (mean age $=24.54 ; \mathrm{SD}=4.14$ ). All had normal or corrected-tonormal visual acuity, French as their mother tongue and reported no language impairments (e.g. dyslexia). None had experienced psychiatric or neurological disease or unexplained loss of consciousness, and none reported having experienced any epileptic episodes in their past or having a history of epileptic events in their family. The experiment was performed with the informed and overt consent of each participant. Participants were paid for their participation $(30 \mathrm{CHF} / \mathrm{h})$ and were free to withdraw from the study at any time.

Results of three participants were excluded from the analysis due to outlier detection levels or a high level of false alarms. Two participants had high levels of false alarms (wrong detection in pseudo-words condition) in all three conditions of TMS, indicating that they had difficulties discriminating words from pseudo-words or were not focused on the task. In both cases, the false alarm levels were considered as outliers according to the mean plus or minus 
two standard deviation criterion. One other participant had extremely high levels of average detection, which also met the criteria for outlier exclusion-i.e. 2 standard deviations above the group mean. The analyses for the study 2 (TMS) were thus conducted on a sample of ten participants.

\section{Stimuli and procedure}

The same simultaneous bilateral lexical decision task as in study 1 (EEG) was used with the same stimuli presented in the same manner and with the same instructions. The only difference with the task from study 1 was that the inter-stimulus interval was randomly jittered from 3,340 to 3,640 ms-i.e. an extended period to avoid cumulative effects of TMS between trials. Responses of the participants were collected during 2,500 ms (Fig. 1).

The task was divided into blocks of 48 trials, freely randomised for each block and for each participant. Eight neutral words in the LVF, eight neutral words in the RVF, eight emotional words in the LVF, eight emotional words in the RVF, and 16 pairs of pseudo-words were presented per block. The day before the TMS experiment, participants were asked to perform four blocks as behavioural measurement and training session without any TMS involvement. Then, on the following day, they had to execute three blocks of the task while TMS was released over each site of stimulation ( 3 blocks $\times 3$ sites of stimulation). In addition, each word was first displayed as single word in a central position below the fixation point and subjects performed a simple word detection task. This was done to avoid learning effects during the task due to the repeated presentation of a word.

\section{Transcranial magnetic stimulation parameters}

The TMS parameters were in accordance with the current safety guidelines of TMS use (Rossi et al. 2009). A Magstim Rapid ${ }^{2}$ with a double 70-mm figure-of-eight coil (The Magstim Company Ltd, Whitland, Wales, UK) was used for magnetic stimulation. TMS was performed as an eventrelated paradigm to interact transiently with cortical areas of interest during the task. A train of three bipolar pulses at $20 \mathrm{~Hz}$ was released $50 \mathrm{~ms}$ after each onset of the stimulus-i.e. the pulses were delivered 50,100, and $150 \mathrm{~ms}$ post-stimulus (Fig. 1). Hence, the TMS pulses are delivered after the display of the letter strings, thus avoiding alerting effects on perception due to sound or skin sensations induced by TMS. The strength of stimulation was set individually to $90 \%$ of the resting motor threshold (RMT) intensity-i.e. the intensity, which generates a finger movement for more than half of the motor cortex stimulations. Reduced intensity is known to produce a more focal effect (Wagner et al. 2009). In addition, a moderate intensity of stimulation limits participant discomfort and muscle contraction.

Both the time period and the sites of stimulation were based on the results of the EEG study (see "EEG-based TMS target choice"). On a different day prior to the TMS sessions, the participants underwent a T1-weighted structural MRI scan. The target sites for TMS stimulation were determined based on individual structural MRI on the anterior bend of the angular gyrus-Brodmann area 39, in the middle of the TPJ of both hemispheres. The average Talairach coordinates of the actual targets across participants were $-58.9 ;-54 ; 25.8$ for the left angular gyrus (LAG) and $58.2 ;-50.7 ; 27.8$ for the right angular gyrus (RAG). The vertex $(0 ;-25 ; 68)$ was used as an active control site. A frameless neuronavigation system combining the neuronavigation module of BrainVoyagerQx (BrainInnovation, Maastricht, the Netherlands) with the ultrasound CMS20 measuring system for navigation (Zebris $\mathrm{GmbH}$, Tübingen, Germany) and based on individual structural MRI was used to place and to maintain the coil over the considered site for every single task block. The three different locations of the stimulation were alternated following a sequence of nine blocks in a single-blind design; sequenced as $\mathrm{a} / \mathrm{b} / \mathrm{c} / \mathrm{a} / \mathrm{b} / \mathrm{c} / \mathrm{a} / \mathrm{b} / \mathrm{c}$. The stimulation sites were randomly attributed to $\mathrm{a}, \mathrm{b}$, or $\mathrm{c}$ between participants. For the two lateralised positions, the direction of the currents flowing through the coil at the junction of the two wings was oriented from superior medial to inferior lateral. In the vertex condition, the currents were oriented forward.

\section{Analysis of behavioural data}

Average accuracy and median RT of correct detections were calculated for each stimulus condition and each TMS condition in each subject. These behavioural data were computed for conditions with real words using a three-way ANOVA with the within-subject factors: TMS-i.e. site of stimulation: LAG versus RAG versus vertex, Lateralityi.e. side of presentation: LVF versus RVF, and Emotionality-i.e. valence of word: neutral versus emotional. Missed events and false alarms were also computed in order to detect potential outlier subjects (not represented).

The accuracy and median RT for the LAG and RAG stimulations were individually normalised by the behavioural variables obtained for the control vertex stimulation as follows:

Vertex normalized index $=$ (variable LAG or RAG

- variable vertex)/(variable LAG or RAG + variable vertex).

The variables thus obtained were vertex normalised indices (VNI). In the case of accuracy, a positive VNI reflects an increase in detection performance caused by TMS over LAG or RAG compared with accuracy obtained after 
vertex stimulation. Concerning RT, a positive VNI indicates a slowing of the response speed. This normalisation reduces unspecific side effects of TMS that could be due to peripheral sensations as well as the alerting properties of the loud click sound of the TMS stimulation (see also Romei et al. 2011; Rochas et al. 2013). Note that this index is unit-free.

The VNI for accuracy and median RT were computed using a three-way ANOVA $(2 \times 2 \times 2)$ with the withinsubject factors: TMS-i.e. site of the TMS: LAG versus RAG, Emotionality-i.e. valence of the word: neutral versus emotional, and Laterality-i.e. side of presentation: LVF versus RVF.

\section{Results}

Study 1 (EEG)

\section{Behavioural results}

Figure 2 indicates the mean and standard error of the accuracy and median RT in the EEG study. In view of our analysis, the results for both accuracy and median RT providing two tests for each effect, a $p$ value $<0.025$ critical level was chosen to maintain an overall $p$ value $<0.05$ error rate. Greenhouse-Geisser corrections were employed for all effects. As can be seen in Fig. 2, both the expected RVF advantage [accuracy: $F_{(1,12)}=12.82 ; p$ value $=0.004$ and RT: $F_{(1,12)}=6.28 ; p$ value $=0.028$ n.s.] and the expected emotional word advantage [accuracy: $F_{(1,12)}=0.49 ; p$ value $=0.50$ n.s. and RT: $F_{(1,12)}=17.09 ; p$ value $\left.=0.001\right]$ were present. An interaction between emotional word advantage and visual field was observed [accuracy: $F_{(1,12)}=31.67$; $p$ value $<0.001$ and RT: $F_{(1,12)}=1.46 ; p$ value $=0.25$ n.s. $]$.

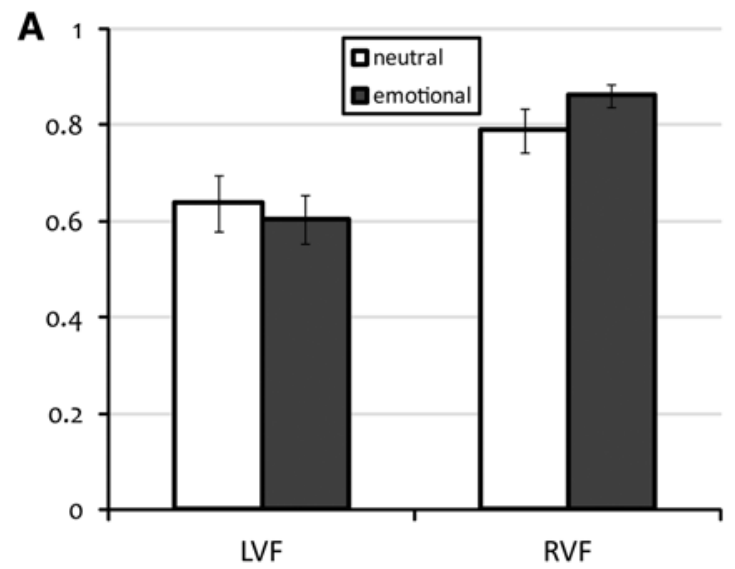

\section{ERP's topographic ANOVA results}

The time-point-by-time-point $T$-ANOVA revealed significant differences for the factor Emotionality from 116 to $134 \mathrm{~ms}$ after the stimulus onset (Fig. 3a). The difference map indicated increased positivity over anterior electrodes for neutral words and increased positivity for emotional words over posterior electrodes for this time window. Based on the global field power of the grand-mean ERPs (not represented), this time period falls in the transition between the P100 component and the subsequent N170like component.

The comparison for the factor Laterality showed differences in a large time window, starting just $52 \mathrm{~ms}$ after stimulus presentation and covering the entire P100 component-from 52 to $140 \mathrm{~ms}$ (Fig. 3b). In this period, the difference map between the LVF and RVF conditions showed a clear left-right polarity inversion with relative stronger right positivity when the real word was detected in the LVF and the pseudo-word in the RVF, and a left positivity in the reversed condition. The corresponding averaged maps during this time period, resembling the visual P100 topography, had left- and right-side maxima of positive potentials that were inverted between the two conditions, with more positivity over the right posterior electrodes for LVF words and more positivity over left posterior electrodes for RVF words. The two conditions differed again in a second time window starting at $184 \mathrm{~ms}$ - from 184 to more than $250 \mathrm{~ms}$. This time, the difference map showed inverted polarities over frontal electrodes with more positivity over right frontal electrodes for words in the LVF and more left frontal positivity for words in the RVF.

There were interactions between the two factors lasting less than $10 \mathrm{~ms}$ at $130 \mathrm{~ms}$, then more durably from 158 to

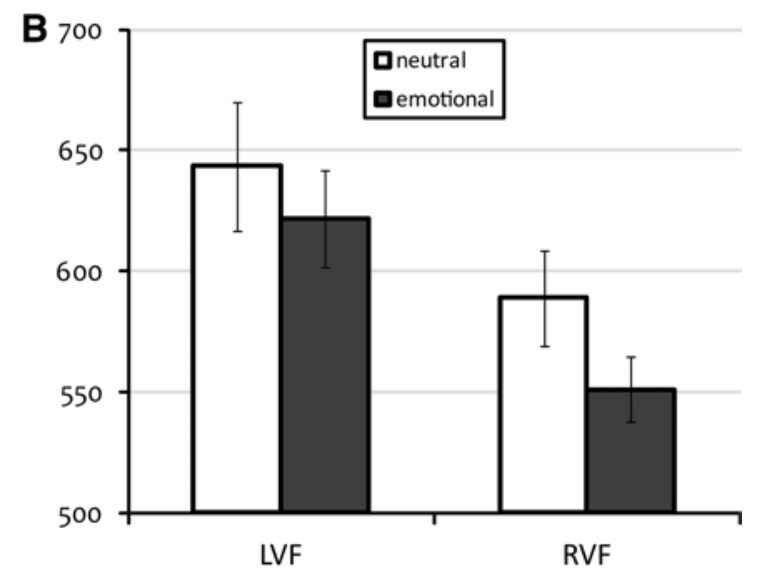

Fig. 2 Averages and standard errors of the individual accuracies (a) and median RT in ms (b) for the four stimulus conditions with words (left or right visual field neutral word, and left or right visual field emotional word) in study 1 (EEG) $(n=13)$ 
Fig. 3 Topographic ANOVA comparisons of data according to the emotional nature (emotionality) or the side of presentation (laterality) of words and their interaction across time from 50 to $250 \mathrm{~ms}$ after the onset of the stimulus. Black solid rectangles depict time points of significance $(p<0.05)$, and the topographic maps $(\mu \mathrm{V})$ show the maps averaged for these time periods for the corresponding conditions as well as the difference maps between them $(n=13)$

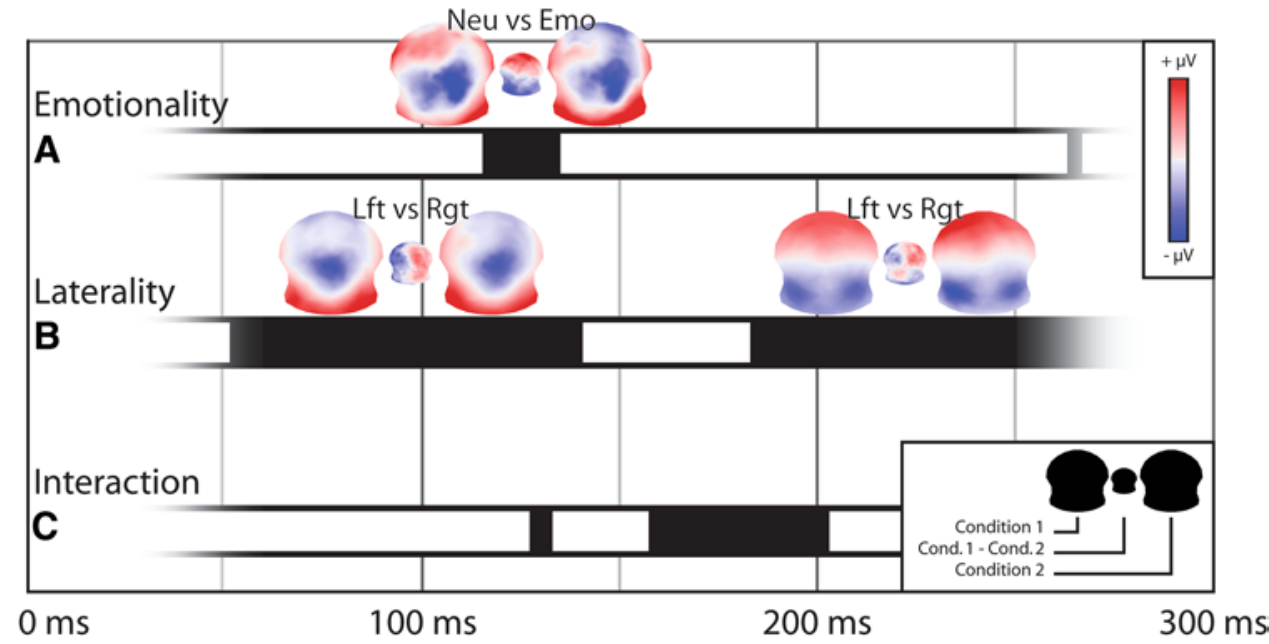

$202 \mathrm{~ms}$ (Fig. 3c). Interactions signified that differences of map configurations for the Emotional factor are dependent on the difference according to the side of presentation; in other words, the differences of processing between neutral and emotional words were dependent on the visual field in which they were presented. The scheme of the interaction opposes any real word in the RVF and neutral words in any visual field against words in the LVF and emotional words; this indicates that words in the RVF share similar brain states with neutral words, while words in the LVF show a similar response to emotional words.

Critically though, the chronology of these effects suggests that map topographies differed first as a function of the side of presentation of the word (50 ms post-onset), then as a function of the emotional content of the words, and finally with an interaction of these two factors after $150 \mathrm{~ms}$. From $210 \mathrm{~ms}$ onwards, the only remaining differences were for the side of word presentation.

\section{Electrical source imaging (ESI) results}

The analysis in the inverse space focused on those time periods that revealed significant topographic differences in the analysis of the scalp maps-i.e. around the P100 component and the N170-like component.

Considering the first period of interest-i.e. from 70 to $134 \mathrm{~ms}$, the analyses of variances on the $z$-scored current density values revealed differences for the main factor Laterality similarly to the scalp map analysis. These differences were found mainly in the right PrecuneusBrodmann area 7 (Fig. 4a), and somewhat weaker in the right inferior parietal lobule and RAG_-Brodmann area 39, and in the left superior and middle temporal gyri-Brodmann areas 21, 22, 41 and 42. Post hoc analysis revealed a stronger activity in these areas for words detected in the LVF as compared to the RVF.
Concerning the second time period of interest-i.e. from 130 to $210 \mathrm{~ms}$, the analysis of variance revealed differences for both main factors Laterality and Emotionality and for the interaction between the two factors. The first differences were found for the factor Emotionality in the very beginning of the period from 132 to $156 \mathrm{~ms}$, in a large cortical area from the right inferior to the superior parietal lobule_-Brodmann areas 7, 19, 39 and 40. Post hoc analyses revealed stronger activity for emotional words than for neutral words in these areas (Fig. 4b). The factor Laterality differed from $148 \mathrm{~ms}$ onwards. From 148 to $170 \mathrm{~ms}$, the differences were found first in the right supramarginal gyrus-Brodmann area 40, and in the left post-central gyrus. Afterwards, from 172 to $200 \mathrm{~ms}$, differences were found in an area connecting the posterior part of the right middle temporal gyrus, the RAG, and the anterior part of the right extrastriate cortex-Brodmann areas 19, 37 and 39, in the left Insula-Brodmann area 13, and in the left inferior frontal gyrus. All these areas of divergence were found to be more active for the detection of words presented in the LVF than in the RVF, the most relevant area being the right middle temporal gyrus (Fig. 4c). Finally, the ANOVA revealed interaction effects at the end of the period from 172 to $206 \mathrm{~ms}$, with differences located in the left precuneus and in the posterior part of the left middle temporal gyrus-Brodmann area 21 (not represented). The interaction was mainly due to stronger activity for emotional words detected in the LVF than neutral words detected in the RVF.

\section{Study 2 (TMS)}

\section{EEG-based TMS target choice}

The targets of the TMS and the timing of stimulation were both based on the results of the EEG study. As described in the EEG results section above, the main differences identified by the $z$-score statistics of ESI were recurrently 

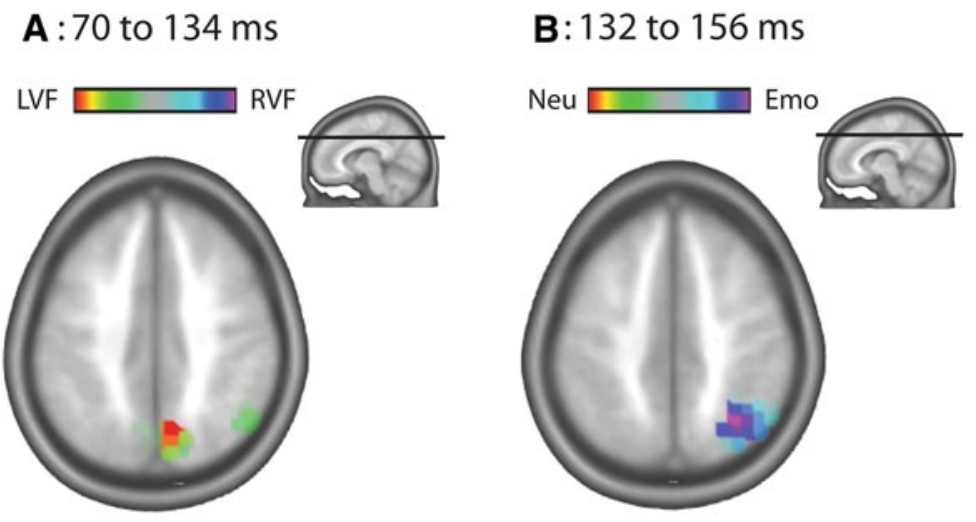

C: 148 to $200 \mathrm{~ms}$

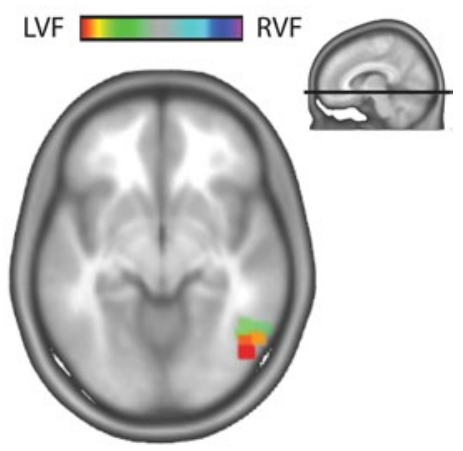

Fig. 4 Graphical representations on horizontal sections of MNI template brain of the significant differences $(p<0.01$ for at least $10 \mathrm{~ms}$ ) from ANOVA of the $z$-scored current density values according to the factors Emotionality (Neu/Emo) and Laterality (LVF/RVF), weighted by the $t$ values from post hoc analyses and averaged over time periods. Warm colours depict differences in favour of the first group

located in the right temporoparietal area during the periods of interest from 70 to $200 \mathrm{~ms}$ (Fig. 4). More specifically, from the beginning of significance of the Emotionality factor at $132 \mathrm{~ms}$, these differences in the right hemisphere were found on a triangle delineated by the posterior part of the right middle temporal gyrus, the right superior parietal lobule and the anterior part of the right extrastriate cortex. Therefore, the right temporoparietal junction was chosen as a target area for TMS and more precisely in the angular gyrus. Interestingly, according to the averages of the $z$-scores of ESI (not represented) and following the initial activation of the primary visual cortices, the left TPJ was consistently activated in any condition while the right was more activated by emotional and LVF words-as suggested by the statistical analysis in ESI. Consequently, we decided to test the implication of the two hemispheres by stimulating two homologous areas in the TMS study. These bilateral activations were present from around $70 \mathrm{~ms}$. We chose to initiate the stimulation at $50 \mathrm{~ms}$ in order to disturb the focused area before the reception of information-i.e. the transfer from the primary visual cortex to the ipsilateral TPJ—and to curtail the propagation to other areas.

\section{Behavioural data and vertex normalised index}

The mean and standard error of the accuracy and median RT are resumed in Table 1 . The volunteers did not report any difficulties executing the task during TMS, and accuracy was above chance $(>0.33)$. In view of our analysis, the results for both accuracy and median RT providing two tests for each effect, a $p$ value $<0.025$ critical level was chosen to maintain an overall $p$ value $<0.05$ error rate.
(Neutral or LVF), and cold colours depict differences in favour of the second group (Emotional or RVF). Brain sections were represented in neurological convention ("left is left"). For an easier reading of the obtained differences, graphical representations were condensed on the maximal difference averaged on time periods of interest based on the topographical results $(n=13)$

Table 1 Averages and standard errors of the individual accuracies and median RT for the four stimulus conditions with words (left or right visual field neutral word, and left or right visual field emotional word) and the three conditions of TMS (vertex, left AG, and right AG) in study 2 (TMS) $(n=10)$

\begin{tabular}{lccccc}
\hline & \multicolumn{2}{l}{ Neutral } & & \multicolumn{2}{l}{ Emotional } \\
\cline { 5 - 6 } \cline { 5 - 6 } & Left & Right & & Left & Right \\
\hline Accuracy & & & & \\
Vertex & & & & \\
Average & 0.479 & 0.783 & 0.525 & 0.825 \\
SE & 0.048 & 0.046 & 0.054 & 0.037 \\
LAG & & & & \\
Average & 0.446 & 0.763 & 0.454 & 0.813 \\
SE & 0.057 & 0.035 & 0.029 & 0.020 \\
RAG & & & & \\
Average & 0.471 & 0.813 & 0.488 & 0.833 \\
SE & 0.049 & 0.043 & 0.055 & 0.032 \\
Median RT & & & & \\
Vertex & & & & \\
Average & 799.6 & 651.8 & 727.9 & 624.2 \\
SE & 32.0 & 37.3 & 40.4 & 37.2 \\
LAG & & & & \\
Average & 782.0 & 678.7 & 735.4 & 637.7 \\
SE & 45.4 & 45.7 & 39.2 & 47.9 \\
RAG & & & & \\
Average & 773.5 & 677.9 & 778.7 & 629.9 \\
SE & 51.3 & 49.9 & 39.5 & 45.0 \\
\hline
\end{tabular}

Greenhouse-Geisser corrections were employed for all effects. As in study 1, the data demonstrated a replication of the well-known advantage for the detection of words in 
Table 2 Averages and standard errors of the vertex normalised index (VNI) of accuracy and median reaction times (RT) for TMS over left and right angular gyri (AG) for the four stimuli conditions with words (left or right visual field neutral word, and left or right visual field emotional word) in the study 2

\begin{tabular}{lrrrrr}
\hline & \multicolumn{2}{l}{ Neutral } & & & \multicolumn{2}{l}{ Emotional } \\
\cline { 6 - 6 } \cline { 5 - 6 } & Left & Right & & Left & Right \\
\hline VNI accuracy & & & & \\
LAG & & & & \\
Average & -0.051 & -0.009 & & -0.053 & -0.005 \\
SE & 0.053 & 0.028 & & 0.045 & 0.020 \\
RAG & & & & \\
Average & 0.039 & 0.029 & & 0.003 & 0.011 \\
SE & 0.064 & 0.019 & & 0.055 & 0.022 \\
VNI median RT & & & & \\
LAG & & & & 0.006 \\
Average & -0.015 & 0.017 & 0.005 & 0.015 \\
SE & 0.019 & 0.013 & 0.022 & \\
RAG & & & & 0.001 \\
Average & -0.023 & 0.015 & 0.034 & 0.007 \\
SE & 0.028 & 0.016 & 0.026 &
\end{tabular}

Positive VNI indicates augmented accuracy or slowed RT compared with vertex stimulation $(n=10)$

the RVF compared with the LVF [accuracy: $F_{(1,9)}=55.26$; $p$ value $<0.0001$ and RT: $F_{(1,9)}=22.77 ; p$ value $\left.=0.001\right]$ and for emotional words compared with neutral words [accuracy: $F_{(1,9)}=0.96 ; p$ value $=0.95$ n.s. and RT: $F_{(1,9)}=10.13 ; p$ value $\left.=0.01\right]$. Neither an interaction nor an effect of the factor TMS was found.

The mean and standard error of VNI for accuracy and median RT are summarised in Table 2. In view of our analysis, the results for both accuracy and median RT providing two tests for each effect, a $p$ value $<0.025$ critical level was chosen to maintain an overall $p$ value $<0.05$ error rate. Greenhouse-Geisser corrections were employed for all effects. Results of the ANOVA with the VNI of accuracy did not show independent effects of a factor or any interaction. The results for the ANOVA with the VNI of the RT also did not show any independent effects of the different factors-i.e. location of TMS, Laterality (side of presentation) or Emotionality. However, there was an interaction between the factors Laterality and Emotionality $\left[F_{(1,9)}=8.02 ; p=0.02\right]$. While the limited statistical power provided by the $n=10$ sample size precludes follow-up post hoc statistical tests of this interaction, the basis for the interaction appears from Fig. 5, the detection of emotional words presented to the LVF was more slowed by TMS in comparison with the detection of neutral words, while the reverse was true for words presented to the RVF.

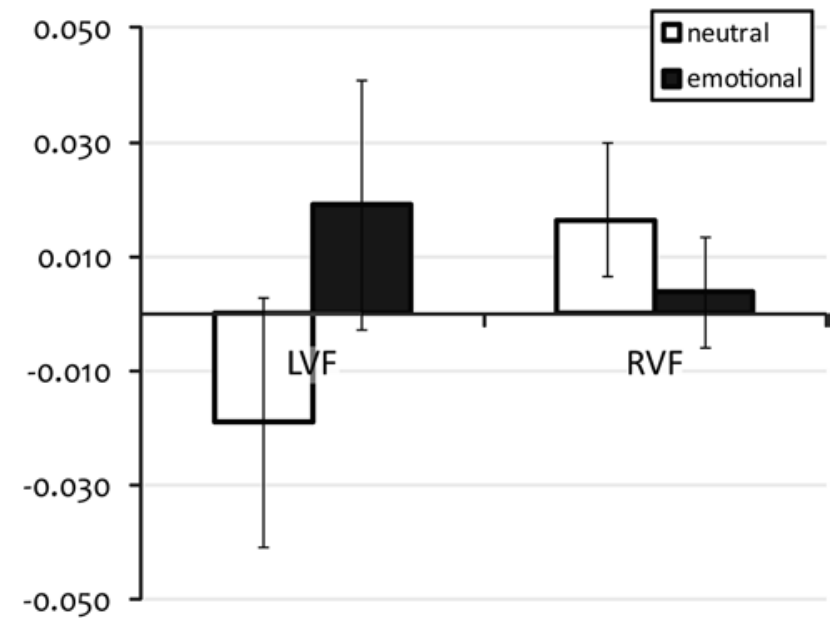

Fig. 5 Averages and standard errors of the vertex normalised index (VNI) of median reaction times (RT) merged between left and right TMS for the four stimulus conditions with words (left or right visual field neutral word, and left or right visual field emotional word) in study 2. A positive VNI indicates slowed RT compared with vertex stimulation $(n=10)$

\section{Discussion}

Performances in word detection change from one individual to another (Levy et al. 2010) and as a function of the stimuli or their environment. Differences of the type of words and the conditions in which they are presented lead to multiple detection strategies reflected also by differences in performance (Riba et al. 2010). The location of the word in the visual field will define by which cerebral hemisphere the visual information is initially treated. The brief simultaneous bilateral presentation used in our experiments allows a single-letter string to be initially projected to its contralateral target hemisphere as segregated information since it excludes reflexive saccades during these very short presentation times. While this dissociation at the entry stage might appear unnatural, the distinction between hemispheres for word detection constitutes an interest for the understanding of the functional lateralisation and the balance between the two hemispheres in the case of challenging situations in the healthy brain or following brain injuries such as stroke. The other factor tested in the present study was the emotionality of the words. Emotionality is known to lead to an advantage in detection compared with neutral conditions and to interfere with the processing of word reading (Schacht and Sommer 2009; Kissler and Koessler 2011; Skrandies 2013). Since a lexical analysis would be assumed to start before a word can be characterised as emotional, this early differentiation for emotional word detection is surprising. The current study confirmed and refined the previous findings on the specificity of processing of emotional words and the role of the right hemisphere in lexical decision 
(Ortigue et al. 2004) by further analyses with new algorithms and extended them by showing that TMS during these early latencies over the temporoparietal areas selectively influences emotional word processing. In addition, it revealed a cooperative engagement of these homologous areas. Our results on specific early bilateral processing of written words will be integrated in a spatiotemporal model of cerebral mechanisms.

\section{Early bilateral processing of words}

The behavioural data show that the participants recognised the words-accuracies were above chance in all conditions for the two studies (Fig. 2; Table 1), denoting non-subliminal phenomena in spite of the rapid presentation of the stimuli. Moreover, behavioural results in the two studies show that our paradigm was in accordance with the well-known advantage for the detection of words presented to the RVF in the right-handed male subject (Young et al. 1980; Graves et al. 1981; Iacoboni and Zaidel 1996; Babkoff et al. 1997) and the advantage for the detection of emotional words compared with neutral words (Landis 2006). For any trial, each visual hemifield, and therefore each opposite hemisphere, receives a four-letter string flashed exactly in the same fashion. The only remaining difference is the significance of these letters-i.e. they are a pseudo-word or a real word, neutral, or emotional. Words are detected better and faster when they are presented in the RVF compared with the LVF, and they are also detected faster when they are emotional. These behavioural results reflect the electrophysiological signals. In spite of apparent similar global topographic maps and electric source images in all conditions-a certain similarity is to be expected when physical parameters are identical between conditions-the inter-condition contrasts stand out with obvious differences. The cerebral processing is already different at early stages, during the P100 component, according to the side of presentation of a word and according to its emotional content. These results agree with a rapid access to the lexical dimension after stimulus onset reported in the previous literature (Scott et al. 2009; Hauk et al. 2009; Segalowitz and Zheng 2009; Skrandies 2013). These differences occur, however, much earlier than the classically reported time for letter string processing around $150 \mathrm{~ms}$ (Grainger and Holcomb 2009; Pylkkanen and Marantz 2003). Moreover, the divergences found in our bilateral lexical decision studies in EEG and TMS argue for a specific detection at early stages in each hemisphere. These early latencies argue for an extremely fast start of the discrimination process before the crossing of the information to the opposite hemisphere is likely. Similar early differences in the ERPs between 80 and $150 \mathrm{~ms}$ depending on the connotative meaning of visually presented words have been described by Skrandies in adults as well as in children (Skrandies 2013). The fact that in our study the words are very short and familiar plays a probable role in the swiftness of processing. Dissimilarities between the detection of a word in the LVF versus in the RVF shown by topographic analysis are mainly reflected as differences in left-right lateralisation of the polarities (Fig. 3b) during the two time windows of interest-i.e. P100 and N170. More than a simple left-right inversion of the pattern of activations in the brain, the ANOVA on the $z$-scored current density values explains this persistent dissimilarity in topography with additional generators initially located in the right precuneus with a supplementary activation for words detected in the LVF (Fig. 4a), in addition to the standard network activated by the detection of words in the RVF. While the P100 mainly corresponds to the processing of visual information in the visual cortex, these results argue for differences that are due to concomitant preprocessing of word material in areas beyond the primary cortex. The precuneus has functional connections with 1) the visual cortex from its posterior part along the parietooccipital fissure and 2) the inferior parietal lobule and the superior temporal sulcus from its central part near the precuneal sulcus (Margulies et al. 2009; Zhang and Li 2012). The expected activation of the visual cortex by words flashed in both hemifields is followed by the activation of the inferior parietal lobule and the superior temporal sulcus for further steps of word processing. Indeed, the divergences are then located in the areas adjacent to the occipital lobe in particular with the right TPJ around $130 \mathrm{~ms}$ and the right middle temporal gyrus at $170 \mathrm{~ms}$ (Fig. 4b, c). Again, the results indicate supplementary activations in the right hemisphere, respectively, for the detection of emotional words and words presented in the LVF.

A delayed but superimposed processing of the emotionality

The assumption of a lexical detection process in the right hemisphere for words presented to the LVF would not have been validated by the isolated analysis of the Laterality factor in the EEG study. Indeed the observed difference between the two conditions, LVF versus RVF, at a given time could be solely due to a detection of a word in the RVF by the left hemisphere versus a non-detection in the other. However, the results from the ANOVA on the $z$-scored current density values indicate functional activations of the right hemisphere. Moreover, the early divergences in the processing of emotionality (Fig. 3a, b) and the interactions involving the factors Emotionality and Laterality point to an actual early detection of words also in the LVF and different detections in both hemispheres. The TMS results argue in favour of a cooperative network between both hemispheres that is balanced according to the two factors. Specific effects for emotional words in the LVF point to a semantic treatment. 
Compared with the Laterality factor, the differences due to the factor Emotionality in the EEG study are more focused in time and appear a little later at $116 \mathrm{~ms}$ in the topography analyses (Fig. 3a)-orthogonally to what was observed in the results for the factor Laterality. Results from the ANOVA on $z$-scores of current density values show brief but strongly significant differences for the factor Emotionality between the P100 and the N170-like components, from $132 \mathrm{~ms}$ onwards, with a maximum of increased activity for the detection of emotional words compared with neutral words in the right TPJ (Fig. 4b). Hence, the emotional differentiation might thus occur downstream of the primary visual cortices. Interestingly, the TPJ is connected with the precuneus and also with the primary visual cortex (Richardson et al. 2011; Zhang and Li 2012).

Additionally, the slight emotional advantage in detection in the RVF in study 1 (Fig. 2) could be due to the observed bilateral implication of the TPJs. Indeed, RVF emotional words could be processed in the left hemisphere as they come from the RVF and by an extra processor in the right hemisphere for their Emotionality (Fig. 4b). Therefore, the two sites involved in RVF emotional word processing might lead to a redundant lexical treatment and reduce missed events while only the right side is involved in the processing of emotional words from the LVF in the conditions of our experiment-i.e. with short, abstract, and very familiar words. Interestingly, the absence of a significant advantage in RT for these emotional words coming from the RVF (Table 1) is in accordance with a model of double processing in the left and the right hemispheres. The bilateral processing of these words would be better but not necessarily faster due to multiple exchanges between brain areas.

\section{Functional interaction between the conditions}

Obviously the brain computes words in the LVF or the RVF as well as neutral or emotional words in a different way. Nevertheless, the interactions found in the EEG and TMS results during the first $250 \mathrm{~ms}$ of processing suggest that word detection varies as a function of Emotionality and Laterality in a dependent and similar way and indicate a difference but not a clear distinction. More precisely, the processing of words in the LVF is related to the processing of emotional words, while the detection of neutral words and of words presented in the RVF would also converge to similar brain states. A possible reason could be that the treatments of these two characteristics interact in the processing of word detection because they share similar structures or networks - as their disruption by short-burst TMS tends to assert. In the case of regular word detection, some unavoidable relays may be used. The interactions would be generated by interdependent collisions (interactions) in these common neural substrates. As developed hereafter, the TPJ regions might be at the centre of a possible common network. As TMS is released from 50 to $150 \mathrm{~ms}$, the physiological functions of the target area and possibly its connected areas are degraded during this time period and the following decades of milliseconds (Valero-Cabré et al. 2007). TMS applied over the left or RAG does not disturb the key tendencies of the behaviour of the participants. The detection is still better for words presented in the RVF compared with LVF, and emotional words are detected faster than neutral words regardless of the TMS conditions (Table 1). However, compared to what is observed for the vertex stimulation, TMS modifies the RT when applied over the left or RAG according to the factors Laterality and Emotionality (Table 2). Nevertheless, as the slowing is not the same for all categories of stimuli, the implication of the angular gyri is stimulus-dependent. Indeed, the behavioural perturbation mediated by the disruption of the angular gyri in the first $200 \mathrm{~ms}$ is an interaction indicating that emotional word detection in the LVF is more impaired by TMS in comparison with the detection of neutral words. This is supported by the difference between neutral and emotional word detection for the LVF, while the detection of words in the RVF shows a weaker difference (Fig. 5). In this sense, the detection of words in the LVF would have a more weight in the interaction found in the ANOVA of VNI for median RT. The TMS evidence indicates that the processing of emotional words in the LVF can be impaired by acting on bi-hemispheric targets of the TPJs, namely the angular gyri. Both TPJs might have cooperative functional roles in word detection as already suggested by differences found in both hemispheres in the ESI analyses. Notably, previous studies using non-repetitive TMS showed rapid contralateral propagations of TMS-evoked activations to the homologous area in the motor cortex (Ilmoniemi et al. 1997; Parks et al. 2012; Casali et al. 2013; Ragazzoni et al. 2013), the premotor cortex (Massimini et al. 2005) as well as the visual cortex (Ilmoniemi et al. 1997). Although all these studies used single-pulse stimulation protocols, a transcallosal propagation with our triple-pulse stimulation that would lead to the observed absence of distinction between left and right TMS cannot be fully ruled out. The right side that is specialised for emotional processing could thus also be disturbed by the stimulation of the left side. In this case, the effect of the TMS of the left TPJ could be due to the impairment of the activations found in the ESI analyses in the right TPJ. While differences between TMS over the left or the right TPJs did not reach statistical significance, the stronger slowing of the detection of emotional words from the LVF due to the disruption of the right TPJ (highest VNI for median RT in Table 2) could result from a main contribution of this structure in the network activated 
by the detection of emotional words and words in the LVF as revealed concordantly in the ESI analyses (Fig. 4). This last point would indeed argue in favour of an effect of TMS over the left TPJ mediated by the propagation of disturbance to the right. Further combined TMS-EEG studies during task execution are needed to fully clarify the mechanisms underlying the observed effects.

\section{A left-right balance}

The results from the EEG and TMS experiments argue for a variable implication of both hemispheres in the detection of written words balanced by the conditional involvement of the right hemisphere according to different conditions. Indeed, the main areas of divergence in the EEG analysis are found in the right hemisphere-in or close to the right TPJ—across both time periods of interest. The TMS results confirm the conditional implication of the right TPJ and suggest a possible cooperation with the left homologous TPJ. Thus, the right hemisphere would be more active for the detection of emotional words and words presented in the LVF. The areas thereby involved in the right hemisphere would be part of the process in addition to the regular network, which is located in the left hemisphere. Conversely, the balance would be all the more in favour of the left hemisphere for neutral words and words coming from the RVF, with a reduced intervention of the right hemisphere during early stages of word processing.

The recruitment of the right hemisphere observed for emotional words is in accordance with an important part of the literature demonstrating the role of the right hemisphere in emotional functions in general (from Gainotti 1972, 2005 with the "right hemisphere hypothesis" to the adapted version of the "valence hypothesis" of Davidson 2001 for the comprehension of emotional information) and more interestingly in the processing of emotional words (Landis 2006). Numerous studies were conducted in aphasic patients showing an advantage for emotional word processing with a lesion of the left hemisphere (Landis et al. 1982, 1983; Reuterskiöld 1991) or specific emotion processing impairments with a right hemispheric lesion (Borod et al. 1992; Lalande et al. 1992; Cicero et al. 1999). In addition to the left lateralised language network, the processing of word-related emotionality is also found in the right hemisphere in healthy participants-e.g. Ortigue et al. 2004; Frühholz et al. 2011; Ponz et al. 2013. Our results argue for supplementary processing performed by the right hemisphere at very early stages. Moreover, this lexical emotion processing in the right hemisphere is dependent on the connection with the left hemisphere. This interhemispheric cooperation could explain paradoxical left-sided processing of emotionality in words observed in some studies (Kissler et al. 2007; Herbert et al. 2008).
The role of the TPJ

The TPJs could be a part of the networks as either a pathway or a processor or both. The fact that the TMS-induced disturbance also includes Emotionality supports an advanced treatment of words in both TPJs or at least in the related upstream areas, and would disagree with a simple pathway of the information that does not involve word processing. The left TPJ is known to be implicated in language comprehension (for review Price 2012). Delimited parts of the TPJ have shown their contribution for specific aspects of visual word processing, such as the supramarginal gyrus in the phonologic dimension of visual word recognition (Stoeckel et al. 2009), the angular gyrus in response to semantic versus phonologic aspects (Binder et al. 1999), or in multimodal processing (Bonner et al. 2013), while the posterior superior temporal gyrus is rather implicated in word sound processing (Buchsbaum et al. 2001). A bilateral involvement is depicted in the STG notably for speech intonation processing, and the right temporal lobe is also more engaged in speech processing during sentence and context comprehension (Vigneau et al. 2011; Diaz and Hogstrom 2011). Bilateral activation of the angular gyri and superior temporal gyri is found in word reading compared to non-words (Kuchinke et al. 2005) or angular gyri alone in high-frequency word reading (Graves et al. 2010). In the present work, the two TPJs engage a real cooperation, which is conditioned by the stimulus features to achieve word reading. The right hemisphere is involved for LVF words and emotional words implying a bilateral processing with an interaction between the two hemispheres as shown by interaction in the ESI results and suggested by TMS. Accordingly, Vigneau et al. (2011) also found an implication of the right hemisphere in an inter-hemispheric manner in association with left homologous areas.

Spatiotemporal model

Based on our studies, a spatiotemporal model may be proposed that would explain the path of information processing in four steps. The information from each visual hemifield reaches each primary visual cortex separately $50 \mathrm{~ms}$ after the stimulus display as revealed by the averaged $z$-scores of ESI. From here, divergences are observed in the processing of word detection with maximal divergences around $100 \mathrm{~ms}$ in the right precuneus and slightly in the right inferior parietal lobules according to Laterality. Average activities in $z$-scores of ESI are already found in both TPJs before $100 \mathrm{~ms}$. As a first step, a precursor of the lexical decision involving the classification of a letter string as a word-or not-is elicited in the receptor hemisphere from 50 to $130 \mathrm{~ms}$. Activity is concentrated around the superior temporal sulci with diverging processing in terms 
of Laterality in the right supramarginal gyrus from $160 \mathrm{~ms}$ onwards and then in the superior temporal gyrus-i.e. higher activity is observed for words detected in the LVF. In the meantime, a precursor of a semantic analysis of the letter strings has to be initiated to enable further computation of their emotional characteristics. The emotional detection appears to start with first differentiations between neutral and emotional words at $130 \mathrm{~ms}$. In this second step, while the TPJs are already engaged in lateralised processing, the influence of Emotionality emerges concomitantly from 110 to $150 \mathrm{~ms}$. The superior part of the right TPJ shows a specific treatment of emotionality from 132 to $156 \mathrm{~ms}$ with a higher activity found in the inferior parietal lobule for emotional words. At this point, the processing seems to be specific in each hemisphere while also integrating information from interhemispheric communication. Indeed, the activation suggests an inter-hemispheric crossover of information-i.e. for emotional words from the RVF. From there, the interaction between the two factors appears at $130 \mathrm{~ms}$ and then from 160 to $210 \mathrm{~ms}$. The interaction could indicate a conflict between the processing of different factors in common areas of the two hemispheres from precuneus to temporal lobes via inferior parietal lobules. The interaction found in the TMS study tends to confirm the scenario of this bilateral communication with the contribution of the left and right TPJ. From $210 \mathrm{~ms}$ onwards, the differences were found for Laterality only in the right TPJ and in the primary visual cortex, followed by activations in the primary visual cortex and the left middle temporal gyrus (not related to the "Results" section). This could imply further computations that are related to the motor response required by the task; however, the ESI would rather suggest a feedback process with activations and differences located in primary visual areas, which might enable integration for further semantic processing. From this point onwards, the processing would eventually continue in the left hemisphere.

\section{Conclusions}

In conclusion, our results show that lexical detection processing starts very early in both hemispheres. A bilateral network is required for the detection of words according to the side of presentation and their emotional content. Moreover, analysis of slowed RT in the TMS experiment indicates that interference with both left and right TPJ results in impaired processing of emotional words that were presented to the LVF. Our research confirms the hypothesis of an early cooperation between the two hemispheres conditioned by word features. The complementarity between the left and the right side of the brain together with their interaction in word processing constitutes a critical point in the understanding of reading in healthy subjects but also in the case of recovery from injury.

Acknowledgments This research was supported by the Swiss National Science Foundation: Grant No. 320030_132967 to Theodor Landis and Grant No. 310030_132952 to Christoph M. Michel. The TMS equipment and the Cartool software are supported by the Center for Biomedical Imaging (CIBM), Geneva and Lausanne, Switzerland. The Cartool software was developed by Denis Brunet from the Center for Biomedical Imaging (CIBM), Geneva and Lausanne, Switzerland. http://sites.google.com/site/fbmlab/cartool. The STEN toolbox (http://www.unil.ch/fenl/Sten) was programmed by Jean-François Knebel, from the Functional Electrical Neuroimaging Laboratory, Lausanne, Switzerland, and is supported by the Center for Biomedical Imaging (CIBM) of Geneva and Lausanne and by the National Center of Competence in Research project "SYNAPSY-The Synaptic Bases of Mental Disease"; Project No. 51AU40_125759. The authors would like to thank Alexis Hervais-Adelman who assisted us with the analysis of selecting a balanced number of epochs between conditions in study 1 (EEG). Special thanks go to Gregor Thut who helped to initiate this project and provided precious input and expertise during all stages of the project.

\section{References}

Alexander DM, Wright JJ (2006) The maximum range and timing of excitatory contextual modulation in monkey primary visual cortex. Vis Neurosci 23:721-728

Babkoff H, Faust M, Lavidor M (1997) Lexical decision, visual hemifield and angle of orientation. Neuropsychologia 35(4):487-495

Binder JR, Frost JA, Hammeke TA, Bellgowan PSF, Rao SM, Cox RW (1999) Conceptual processing during the conscious resting state: a functional MRI study. J Cogn Neurosci 11(1):80-93

Bonner MF, Peelle JE, Cook PA, Grossman M (2013) Heteromodal conceptual processing in the angular gyrus. NeuroImage 71:175-186

Borod JC, Andelman F, Obler LK, Tweedy JR, Welkowitz J (1992) Right hemisphere specialization for the identification of emotional words and sentences: evidence from stroke patients. Neuropsychologia 30:827-844

Broca P (1861) Remarques sur le siège de la faculté du langage articulé, suivies d'une observation d'aphémie (perte de la parole). Bulletins de la Société Anatomique de Paris 6:330-357

Brunet D, Murray MM, Michel CM (2011) Spatiotemporal analysis of multichannel EEG: CARTOOL. Comput Intell Neurosci 2011:813870

Buchsbaum BR, Hickok G, Humphries C (2001) Role of left posterior superior temporal gyrus in phonological processing for speech perception and production. Cogn Sci 25(5):663-678

Casali AG, Gosseries O, Rosanova M, Boly M, Sarasso S, Casali KR, Casarotto S, Bruno M-A, Laureys S, Tononi G, Massimini M (2013) A theoretically based index of consciousness independent of sensory processing and behavior. Sci Transl Med 5(198):198ra105

Cicero BA, Borod JC, Santschi C, Erhan HM, Obler LK, Agosti RM, Welkowitz J, Grunwald IS (1999) Emotional versus nonemotional lexical perception in patients with right and left brain damage. Neuropsychiatry Neuropsychol Behav Neurol 12:255-264

Davidson RJ (2001) Prefrontal and amygdala contributions to emotion and affective style. In: Gainotti G (ed) Handbook of neuropsychology, emotional behaviour and its disorders, vol 5, 2nd edn. Elsevier, Amsterdam, pp 111-112

Diaz MT, Hogstrom LJ (2011) The influence of context on hemispheric recruitment during metaphor processing. J Cogn Neurosci 23(11):3586-3597 
Frühholz S, Jellinghaus A, Herrmann M (2011) Time course of implicit processing and explicit processing of emotional faces and emotional words. Biol Psychol 87(2):265-274

Gainotti G (1972) Emotional behavior and hemispheric side of the lesion. Cortex 8(1):41-55

Gainotti G (2005) Emotions, unconscious processes and the right hemisphere. Neuro-Psychoanal 7:71-81

Gazzaniga MS (2005) Forty-five years of split-brain research and still going strong. Nat Rev Neurosci 6(8):653-659

Gazzaniga MS, Hillyard SA (1971) Language and speech capacity of the right hemisphere. Neuropsychologia 9:273-280

Gazzaniga MS, Sperry RW (1967) Language after section of the cerebral commissures. Brain 90:131-148

Grainger J, Holcomb PJ (2009) Watching the word go by: on the time-course of component processes in visual word recognition. Lang Linguist Compass 3(1):128-156

Grave de Peralta Menendez R, Gonzalez Andino S, Lantz G, Michel CM, Landis T (2001) Noninvasive localization of electromagnetic epileptic activity I Method descriptions and simulations. Brain Topogr 14(2):131-137

Grave de Peralta Menendez R, Murray MM, Michel CM, Martuzzi R, Gonzalez Andino SL (2004) Electrical neuroimaging based on biophysical constraints. Neuroimage 21(2):527-539

Graves R, Landis T, Goodglass H (1981) Laterality and sex differences for visual recognition of emotional and non-emotional words. Neuropsychologia 19(1):95-102

Graves WW, Desai R, Humphries C, Seidenberg MS, Binder JR (2010) Neural systems for reading aloud: a multiparametric approach. Cereb Cortex 20(8):1799-1815

Hauk O, Pulvermuller F, Ford M, Marslen-Wilson WD, Davis MH (2009) Can I have a quick word? Early electrophysiological manifestations of psycholinguistic processes revealed by eventrelated regression analysis of the EEG. Biol Psychol 80(1):64-74

Herbert C, Junghöfer M, Kissler J (2008) Event related potentials to emotional adjectives during reading. Psychophysiology 45:487-498

Iacoboni M, Zaidel E (1996) Hemispheric independence in word recognition: evidence from unilateral and bilateral presentations. Brain Lang 53(1):121-140

Ilmoniemi RJ, Virtanen J, Ruohonen J, Karhu J, Aronen HJ, Näätänen R, Katila T (1997) Neuronal responses to magnetic stimulation reveal cortical reactivity and connectivity. Neuroreport 8(16):3537-3540

Kissler J, Koessler S (2011) Emotionally positive stimuli facilitate lexical decisions-an ERP study. Biol Psychol 86(3):254-264

Kissler J, Herbert C, Peyk P, Junghöfer M (2007) Buzzwords. Early cortical responses to emotional words during reading. Psychol Sci 18:475-480

Koenig T, Kottlow M, Stein M, Melie-García L (2011) Ragu: a free tool for the analysis of EEG and MEG event-related scalp field data using global randomization statistics. Comput Intell Neurosci 2011:938925

Koenig T, Stein M, Grieder M, Kottlow M (2013) A tutorial on datadriven methods for statistically assessing ERP topographies. Brain Topogr (Epub 29 Aug 2013)

Kuchinke L, Jacobs AM, Grubich C, Võ ML, Conrad M, Herrmann $M$ (2005) Incidental effects of emotional valence in single word processing: an fMRI study. Neuroimage 28(4):1022-1032

Lalande S, Braun CM, Charlebois N, Whitaker HA (1992) Effects of right and left hemisphere cerebrovascular lesions on discrimination of prosodic and semantic aspects of affect in sentences. Brain Lang 42(2):165-186

Landis T (2006) Emotional words: what's so different from just words? Cortex 42(1):170-179

Landis T, Graves R, Goodglass H (1982) Aphasic reading and writing: possible evidence for right hemisphere participation. Cortex 18:105-112
Landis T, Regard M, Graves R, Goodglass H (1983) Semantic paralexia: a release of right hemisphere function from left hemispheric control? Neuropsychologia 21:359-364

Lehmann D, Skrandies W (1980) Reference-free identification of components of checkerboard-evoked multichannel potential fields. Electroencephogr Clin Neurophysiol 48:609-621

Levy T, Walsh V, Lavidor M (2010) Dorsal stream modulation of visual word recognition in skilled readers. Vis Res 50(9):883-888

Lidzba K, Schwilling E, Grodd W, Krägeloh-Mann I, Wilke M (2011) Language comprehension vs. language production: age effects on fMRI activation. Brain Lang 119(1):6-15

Margulies DS, Vincent JL, Kelly C, Lohmann G, Uddin LQ, Biswal BB, Villringer A, Castellanos FX, Milham MP, Petridesi M (2009) Precuneus shares intrinsic functional architecture in humans and monkeys. Proc Natl Acad Sci 106(47):20069-20074

Massimini M, Ferrarelli F, Huber R, Esser SK, Singh H, Tononi G (2005) Breakdown of cortical effective connectivity during sleep. Science 309(5744):2228-2232

Michel CM, Murray MM (2011) Towards the utilization of EEG as a brain imaging tool. Neuroimage 61:371-385

Michel CM, Murray MM, Lantz G, Gonzalez S, Spinelli L, Grave de Peralta R (2004) EEG source imaging. Clin Neurophysiol 115(10):2195-2222

Michel CM, Koenig T, Brandeis D, Gianotti LRR, Wackermann J (2009) Electrical neuroimaging. Cambridge University Press, Cambridge

Mohr C, Michel CM, Lantz G, Ortigue S, Viaud-Delmon I, Landis T (2005) Brain state-dependent functional hemispheric specialization in men but not in women. Cereb Cortex 15:1451-1458

Mohr B, Endrass T, Hauk O, Pulvermüller F (2007) ERP correlates of the bilateral redundancy gain for words. Neuropsychologia 45(9):2114-2124

Morand S, Thut G, Grave de Peralta R, Clarke S, Khateb A, Landis T, Michel CM (2000) Electrophysiological evidence for fast visual processing through the human koniocellular pathway when stimuli move. Cereb Cortex 10:817-825

Murray MM, Brunet D, Michel CM (2008) Topographic ERP analyses: a step-by-step tutorial review. Brain Topogr 20:249-264

New B, Pallier C, Ferrand L, Matos R (2001) Une base de données lexicales du français contemporain sur internet: LEXIQUE. L'Année Psychologique 101:447-462

Ortigue S, Michel CM, Murray MM, Mohr C, Carbonnel S, Landis T (2004) Electrical neuroimaging reveals early generator modulation to emotional words. Neuroimage 21(4):1242-1251

Papagno C, Fogliata A, Catricalà E, Miniussi C (2009) The lexical processing of abstract and concrete nouns. Brain Res 1263:78-86

Parks NA, Maclin EL, Low KA, Beck DM, Fabiani M, Gratton G (2012) Examining cortical dynamics and connectivity with simultaneous single-pulse transcranial magnetic stimulation and fast optical imaging. Neuroimage 59(3):2504-2510

Pell MD (2007) Reduced sensitivity to prosodic attitudes in adults with focal right hemisphere brain damage. Brain Lang 101(1):64-79

Perrin F, Pernier J, Bertrand O, Giard MH, Echallier JF (1987) Mapping of scalp potentials by surface spline interpolation. Electroencephalogr Clin Neurophysiol 66:75-81

Ponz A, Montant M, Liegeois-Chauvel C, Silva C, Braun M, Jacobs AM, Ziegler JC (2013) Emotion processing in words: a test of the neural re-use hypothesis using surface and intracranial EEG. Soc Cogn Affect Neurosci. doi:10.1093/scan/nst034. (Advance online publication)

Price CJ (2012) A review and synthesis of the first 20 years of PET and fMRI studies of heard speech, spoken language and reading. Neuroimage 62(2):816-847

Pylkkanen L, Marantz A (2003) Tracking the time course of word recognition with MEG. Trends Cogn Sci 7(5):187-189 
Ragazzoni A, Pirulli C, Veniero D, Feurra M, Cincotta M, Giovannelli F, Chiaramonti R, Lino M, Rossi S, Miniussi C (2013) Vegetative versus minimally conscious states: a study using TMS-EEG, sensory and event-related potentials. PLoS One 8(2):e57069

Reuterskiöld C (1991) The effects of emotionality on auditory comprehension in aphasia. Cortex 27(4):595-604

Riba J, Heldmann M, Carreiras M, Munte TF (2010) A neuroimaging study of conflict during word recognition. NeuroReport 21(11):741-745

Richardson FM, Seghier ML, Leff AP, Thomas MSC, Price CJ (2011) Multiple routes from occipital to temporal cortices during reading. J Neurosci 31(22):8239-8247

Rochas V, Gelmini L, Krolak-Salmon P, Poulet E, Saoud M, Brunelin J, Bediou B (2013) Disrupting pre-SMA activity impairs facial happiness recognition: an event-related TMS study. Cereb Cortex 23(7):1517-1525

Romei V, Driver J, Schyns PG, Thut G (2011) Rhythmic TMS over parietal cortex links distinct brain frequencies to global versus local visual processing. Curr Biol 21(4):334-337

Ross ED, Edmondson JA, Seibert GB, Homan RW (1988) Acoustic analysis of affective prosody during right-sided Wada test: a within-subjects verification of the right Hemisphere's role in language. Brain Lang 33(1):128-145

Rossi S, Hallett M, Rossini PM, Pascual-Leone A, The Safety of TMS Consensus Group (2009) Safety, ethical considerations, and application guidelines for the use of transcranial magnetic stimulation in clinical practice and research. Clin Neurophysiol 120(12):2008-2039

Schacht A, Sommer W (2009) Time course and task dependence of emotion effects in word processing. Cogn Affect Behav Neurosci 9(1):28-43

Scott GG, O'Donnell PJ, Leuthold H, Sereno SC (2009) Early emotion word processing: evidence from event-related potentials. Biol Psychol 80(1):95-104

Segalowitz SJ, Zheng X (2009) An ERP study of category priming: evidence of early lexical semantic access. Biol Psychol 80(1):122-129

Skrandies W (2013) Electrophysiological correlates of connotative meaning in healthy children. Brain Topogr (Epub 22 Aug 2013)
Sperry RW (1961) Cerebral organization and behavior. Science 133:1749-1757

Spinelli L, Gonzalez Andino S, Lantz G, Seeck M, Michel CM (2000) Electromagnetic inverse solutions in anatomically constrained spherical head models. Brain Topogr 13:115-126

Springer S, Deutsch G (2004) Left brain, right brain: perspectives from cognitive neuroscience. Prószynski I S-ka SA, Warsaw

Stoeckel C, Gough PM, Watkins KE, Devlin JT (2009) Supramarginal gyrus involvement in visual word recognition. Cortex 45(9):1091-1096

Stroobant N, Buijs D, Vingerhoets G (2009) Variation in brain lateralization during various language tasks: a functional transcranial Doppler study. Behav Brain Res 199(2):190-196

Thorpe SJ (2009) The speed of categorization in the human visual system. Neuron 62(2):168-170

Valero-Cabré A, Payne BR, Pascual-Leone A (2007) Opposite impact on ${ }^{14} \mathrm{C}$-2-deoxyglucose brain metabolism following patterns of high and low frequency repetitive transcranial magnetic stimulation in the posterior parietal cortex. Exp Brain Res 176(4):603-615

Vigneau M, Beaucousin V, Hervé P-Y, Jobard G, Petit L, Crivello F, Mellet E, Zago L, Mazoyer B, Tzourio-Mazoyer N (2011) What is right-hemisphere contribution to phonological, lexico-semantic, and sentence processing? Insights from a meta-analysis. Neuroimage 54(1):577-593

Wagner T, Rushmore J, Eden U, Valero-Cabré A (2009) Biophysical foundations underlying TMS: setting the stage for an effective use of neurostimulation in the cognitive neurosciences. Cortex 45:1025-1034

Wernicke C (1874) Der aphasische Symptomencomplex: Eine Psychologische Studie auf anatomischer Basis. Max Cohn \& Weigert, Breslau

Woody CD (1967) Characterization of an adaptive filter for the analysis of variable latency neuroelectric signals. Med Biol Eng 5:539-553

Young AW, Bion PJ, Ellis AW (1980) Studies toward a model of laterality effects for picture and word naming. Brain Lang 11:54-65

Zhang S, Li CR (2012) Functional connectivity mapping of the human precuneus by resting state fMRI. Neuroimage 59(4):3548-3562 